\title{
Reviews
}

\section{Polycystic ovarian syndrome in infertility}

\author{
Ameet Patki ${ }^{1}$ \\ Sri Lanka Journal of Obstetrics and Gynaecology 2012; 34: 112-119
}

\section{Introduction}

Polycystic ovarian syndrome (PCOS) is a condition of unexplained hyperandrogenic chronic anovulation that most likely represents a heterogenous disorder. About $10 \%$ of women in the reproductive age group suffer from this disorder. Many adolescent girls and young adults seek consultation because of this disorder. It's etiology is as yet undefined, and therapy remains largely symptomatic and empirical. PCOS is associated with metabolic sequelae including risk of diabetes and cardiovascular disorders and hence needs attention to these sequlae. Till 2003 diagnostic criteria for PCOS were varied and often confusing. However the Joint European Society of Human Reproduction and Embryology and The American Society for Reproductive Medicine ESHRE/ASRM sponsored consensus meeting in Rotterdam in 2003 accepted that PCOS consists of the presence of any 2 of the 3 criteria. Oligo- and/or anovulation, clinical and /or biochemical evidence of hyperandrogenism and the presence of polycystic ovaries on ultrasound examination. Other etiologies like congenital adrenal hyperplasia, Cushing's syndrome, and androgen secreting tumours need to be excluded ${ }^{1}$. Ultrasonographic scanning of women with unexplained hyperandrogenic chronic anovulation frequently reveal ovaries that are polycystic ${ }^{2}$. Sonography shows ovarian enlargement, multiple (>10 $\mathrm{mm})$ small follicles at the periphery with increased echogenicity. However polycystic ovaries are a non-specific finding often noted in women with no endocrine or metabolic abnormalities.

Incidence: The incidence is influenced by the nature of the population being assessed. Studies in which ultrasound examination for presence of polycystic ovaries were conducted reveal a prevalence rate of approximately 20 to $30 \%$ in Caucasian women in the reproductive age ${ }^{3}$. There are significant racial difference, and as, compared to white population the prevalence of PCOS is found to be higher in the ethnic groups. In the South Asians living in the UK the prevalence reported was $52 \%{ }^{4}$.

\footnotetext{
${ }^{1}$ Consultant Obstetrician and Gynaecologist, Sir Harkisondas Hospital and Research Centre, Mumbai, India.
}

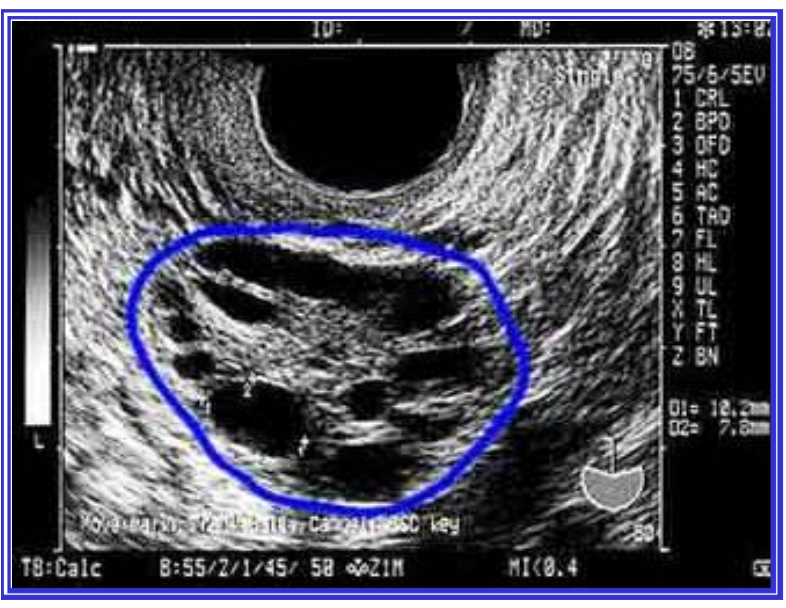

Figure 1. USG showing polycystic ovary.

Etiology: Genetic studies have demonstrated a link between PCOS and metabolic disturbances such as disordered insulin metabolism indicating that the PCOS may be a manifestation of a complex genetic disorder. No gene or specific environmental substance has been identified. Selective insulin resistance may be central to the etiology of PCOS . Thus compensatory hyperinsulinaemia may result in decreased levels of serum hormone binding globulin (SHBG), and serve as a trophic stimulus to androgen production in the adrenals and ovaries and have a direct effect on the hypothalamus causing an abnormally stimulated appetite and increased gonadotropin secretion.

Presently, the view held for the occurrence of PCOS attributes it to enhanced serine phosphorylation unification theory which in turn leads to increased CYP17 activity in the ovary (hyperandrogenism) and reduced insulin receptor activity peripherally (insulin resistance) leading in turn to the endocrine dysfunction of PCOS ${ }^{6}$.

\section{Normal ovulation requirements:}

- Competent hypothalamic-pituitary-ovarian axis

- Coordinated activities of gonadotropins, steroids, inhibin, activin, insulin-like growth factor and their binding proteins, proteases and prostaglandins. 
Pathophysiology: Until date there is no accepted theory as to the pathogenesis of PCOS. Because of the diverse nature of the findings, it is unlikely that any single factor will explain the entire gamut of changes associated with PCOS. It appears more plausible that many factors trigger the occurrence of PCOS, some more common than others. Once established, there is a well described chronicity and self perpetuating mechanism of the syndrome. It is clear therefore that PCOS is a lifelong situation in which possibly genetic factors, endocrine dysfunction and metabolic sequelae play a significant role. The PCOS state may be temporarily interrupted by hormonal therapy, pregnancy or ovarian ablative surgery. An important advance in our understanding of this syndrome has been the realization of the pivotal role of insulin resistance in perpetuating the PCOS status. Insulin resistance represents a unifying component of the syndrome that is present as a common denominator not only in obese PCOS, but also in adolescent girls with hyperandrogenism, in women with multi-follicular ovaries, and in apparently normal women with polycystic ovaries. The main characteristic of insulin resistance in PCOS is that it is a partial resistance which involves metabolic activities of insulin but does not prevent insulin effect on its receptors on the ovary, or the effect on insulin SHBG and the hepatic production of insulin growth factor-1 (IGF-1). The main effect of insulin on the ovaries is not only to increase androgen production, but also to derange the regulation of androgen synthesis so as to prevent the down regulation of the LH receptors leading to increased production of androgens and estrone which coupled with insulin effect of lowering SHBG leads to hyperestrogenism and $\downarrow$ FSH levels.

A genetic basis for the development of PCOS has been suspected, however despite the high prevalence of PCOS within families, no clear cut mode of inheritance has been established.

\section{Summary of endocrine dysfunction:}

- Hypersecretion / Elevation of LH

- Hyperinsulinemia

- Androgen excess

- Disturbed hypothalamic - pituitary - ovarian axis

- Ovarian hyperthecosis

- High levels of intraovarian androgens

- Follicular atresia

- Anovulation

- Menstrual irregularity

- Infertility.
Clinical Manifestations: These women commonly present with infertility or menstrual disturbances like oligomenorrhoea and amenorrhoea, obesity and hirsutism.

A great deal of attention has been focused on the risks of ovulation induction in these women with PCOS because of the enhanced risks of ovarian hyperstimulation, multiple pregnancy, and first trimester pregnancy losses. During pregnancy there is an increased risk for gestation diabetes, hypertension, and preterm birth particularly in the presence of multiple gestation ${ }^{7,8}$. Chronic anovulation, obesity, carbohydrate intolerance, hyperinsulinaemia and decreased levels of SHBG are all associated with endometrial cancer in later years ${ }^{9}$. Hence the rational approach should be to improve the carbohydrate metabolism and thus reduce the endogenous production of excess of insulin. Metformin is effective in improving the glucose metabolism by multivarious approaches, and thus revert the PCOS subject to normal insulinaemic status. Once the axis has been down regulated, the hyperandrogenemia is effectively controlled permitting regular ovulation and conception. Hence there is absence of ovarian hyperstimulation, less vigilante ovulation monitoring, simplicity of drug administration and improved costeffectiveness of therapy. Further, there is a reduction in rates of pregnancy complications and an increase in the take home baby rate.

Women and particularly obese women with hyperinsulinaemia are at an increased risk of developing hypertension, dyslipidaemia and impaired glucose tolerance and type II diabetes meillutus. This cluster of symptoms being referred to as the metabolic $X$ syndrome may increase the risk of cardiovascular disease in later life.

Differential diagnosis: The clinician must consider several possibilities including -

- Exogenous androgens

- Nonclassical adrenal hyperplasia

- Androgen secreting tumours

- Cushing's syndrome

- Acromegaly

- Genetic defects in insulin action

- Primary hypothalamic amenorrhoea

- Primary ovarian failure

- Thyroid dysfunction

- Prolactin disorders. 


\section{Diagnostic evaluation and work-up:}

\section{- Routine physical examination:}

- $\quad$ BMI (Body Mass Index) - 25-30 is considered overweight, $>30$ is obese.

- Waist-Hip Ratio to determine body fat distribution. Values $>0.72$ are abnormal.

- Presence of stigmata of hyperandrogenism or insulin resistance - acne, hirsutism, androgenic alopecia.

- $\quad$ Blood pressure recording

\section{Laboratory investigations:}

- Demonstration of biochemical hyperandrogenaemia.

- Total testosterone $(>200 \mathrm{ng} / \mathrm{dL}) / \downarrow$ Free testosterone $>2.2 \mathrm{pg} / \mathrm{mL}$.

- S. Estradiol and FSH estimations.

- Exclude hypogonadotropic hypogonadism $\left(\downarrow \mathrm{E}_{2}, \downarrow\right.$ FSH).

- Exclude premature ovarian failure $\left(\downarrow E_{2}\right.$, and 个FSH).

- S. Dehydroepiandrosterone sulfate.

- Not of value if S. Testosterone is normal

- Values $>430 \mu \mathrm{g} / \mathrm{dL}$ significant. It isindicative of adrenal source of androgens.

- Levels $>700 \mu \mathrm{g} / \mathrm{mL}$ suggestive of androgen producing adrenal tumour.

- 24 hours urinary cortisol

- Cortisil < $50 \mu \mathrm{g} / 24$ hours

- Exclude Cushing's syndrome if patient is hypertensive.

- Exclusion of other causes of hyperandrogenism.

- Estimation of TSH to exclude thyroid dysfunction

- Estimation of serum prolactin to exclude hyperprolactinemia

- Estimation of $17 \alpha$ hydroxyprogesterone. Non classical congenital adrenal hyperplasia caused by 21-hydoxylase deficiency.

Random normal level $<4$ ng/ml. Fasting $<2 \mathrm{ng} / \mathrm{ml}$.

- Consider screening for Cushing's syndrome, and

- Rare conditions like acromegaly.

- Evaluation for metabolic syndrome X.

Pelvic ultrasonography: PCOS is characterized by the following observations

- Bilateral enlargement of the ovaries $>8.0 \mathrm{~cm}$. Increased volume.
- Thickened tunica albugenea

- Multiple small cysts ( $\geq 12$ foliclles) of 0.2-0.9 cms. in each ovary

- Absence of dominant follicle

- Thickened stroma (hyperthecosis)

- Resting or follicular endometrium

Treatment of polycystic ovary syndrome:

Goals:

- Prevent endometrial hyperplasia, atypia/ cancer

- Restore normal ovulation / fertility

- Restore normal menstruation

- Correct hirsutism

(1). Women with PCOS concerned with menstrual disturbances - desirous of contraception - The commonest medication prescribed in practice is the O.C.Pill.

Oral contraceptive pills: Oral contraceptive pills inhibit LH, diminish the circulating levels of androgens and increase the levels of circulating SHBG. It is advisable to use a low dose combination pill containing low dose of synthetic estrogen in combination with a low-androgenic progestin. Oral pills also reduce DHEAS levels. If these levels are only mildly elevated $(<4.0 \mathrm{ng} / \mathrm{ml})$, OC pills alone will suffice.

(2). Women with PCOS concerned with hirsutism in whom the above treatment fails to give satisfactory results, other modes of treatment need consideration. In addition to cosmetic therapy which gives instanteous effect, anti androgenic drugs like cyproterone acetate along with ethinyl estradiol similar to a COC pill will give in addition to the contraceptive effect, decrease in the hirsuitscores. However therapy will need to be continued for atleast 12 months to have a 50\% reduction in the hirsuitism. Spironolactone 50 - $100 \mathrm{mg}$ b.i.d. may be prescribed to decrease androgenic action on target organs.

GnRH Analogues: These have also been shown to inhibit ovarian androgen production. In combination with oral contraceptive pills, these medications help 1) To prevent hypoestrogenic side-effects. 2) Elevate SHBG levels. 3) Reduce adrenal androgen production. This therapy is expensive and should be reserved for women who do not respond to the above therapies. and are desirous of preserving ovarian function.

(3). Women with PCOS desirous of fertility are treated mostly for anovulatory infertility. A large majority of these women have normal FSH and estradiol levels (WHO Group II). Specialist would 
have to follow a basic principle which is to initially optimize the women's health prior to treatment for infertility with ovulation inducing agents. Treatment should further be directed towards minimizing ovarian hyperstimulation and multiple pregnancies. In obese women, weight reduction improves the chances of spontaneous and drug induced ovulation ${ }^{10}$.

Clomiphene citrate (CC) is the first line treatment in women with PCOS and anovulatory infertility. It is generally started at a dose of $50 \mathrm{mg} /$ day starting from day 2 - day 6 of the period. The dose can be increased upto $100 \mathrm{mg}$ / day. Increasing the dose beyond 100mg/ day is not effective in most cases. Further it causes increasing cervical mucus thickening and also exhibits its anti-estrogenic effect on the endometrial lining; thereby negating any benefits of ovulation should it occur. On CC, the ovulation rate is around $70-80 \%$. However the pregnancy rate is only $30-40 \%$ over six cycles. This disparity between ovulation rate and pregnancy rate may be due to the detrimental effect on the endometrium, the cervical mucus or the further increase in the already elevated LH levels which could preclude pregnancy (Homburg 1998). Clomiphene resistant women (20 to $25 \%$ of anovulatory women) are those who do not respond to CC even in higher doses. These non responders are generally obese with insulin resistance and hyperandrogenemia. Clomiphene failures are those who ovulate but do not get pregnant in six ovulatory cycles. Presently in most countries, $\mathrm{CC}$ is licensed for use for 6 months because of a possible link between long term use ( 12 months and more) and development of ovarian cancer ${ }^{11}$.

Women with a BMI of $>25 \mathrm{~kg} / \mathrm{m}^{2}$, the addition of metformin has shown to enhance ovulation and pregnancy rates. It is used as a single agent or in combination with CC or gonadotrophins. Metformin use is continued in early pregnancy atleast upto the first trimester when it seems to be safe and reduce the rate of early pregnancy loss associated with the syndrome ${ }^{3}$.

There are good studies to indicate that letrozole, an aromatse inhibitor, is an effective agent for induction of ovulation in women with PCOS. Aromatse inhibitors do not demonstrate the adverse peripheral anti-estrogenic effects of CC. However caution needs to be demonstrated in its use due to the possible teratogenicity which needs further evaluation ${ }^{12}$.

CC resistant or CC failure patients need to be treated with gonadotrophins as a sole agent or in combination with oral ovulation drugs. Laparoscopic ovarian diathermy is also an effective option ${ }^{13}$.

Gonadotrophins in treatment: Treatment with gonadotrophins need to be started with low doses. Careful and serial transvaginal monitoring and intermittent serum estradiol measurements will help to reduce hyperstimulation and multiple pregnancy. A chronic low dose step up regime starting with either $37.5-50 \mathrm{IU}$ of recombinant FSH (rFSH) is advised with a view of monofollicular ovulation. The dose can be increased in same amounts after 7 days if there is no response. HCG is used as a trigger in a dose of 500010000 IU. In cases of timed intercourse or intra uterine insemination (IUI), there should not be more than 3 follicles of $18 \mathrm{~mm}$ at trigger. The cumulative conception rates with gonadotropophins are around $60-70 \%$ after 6 to 12 months of treatment ${ }^{3}$. Gonadotrophin (GnRH) agonists are used concomitant with gonadotrophins when the response is significantly increased. As regards GnRH antagonists there is data to suggest use in PCOS where OHSS is reduced or with low dose rFSH for low stimulation protocols. Laparoscopic ovarian drilling (LOD) or laparoscopic electrocoagulation of ovarian surface (LEOS) is reserved for women with PCOS who do not respond well to clomiphene. Following the procedure, spontaneous ovulation is known to occur frequently, and the subsequent response to ovulation inducing agents shows a marked improvement.

Insulin sensitizing agents: Drugs initially developed to treat type 2 diabetes have been used to treat PCOS. Metformin belongs to the group of drugs that improve peripheral insulin sensitivity by decreasing the circulating insulin levels. As these drugs do not enhance insulin production, therefore they are free from the risks of hypoglycaemia. Improving insulin sensitivity is associated with improved ovulation rates, improved glucose tolerance and $\uparrow$ SHBG leading to $\downarrow$ bioavailibility of androgens. The effect of metformin on endometrial hyperplasia is not known. Short term treatments of 3-6 months of metformin in PCOS suggests improvement in ovulatory functions in almost 50\% cases and circulating androgens register a fall. An $\mathrm{NIH}$ sponsored trial demonstrated that metformin can prevent diabetes in a high risk population with impaired glucose tolerance. IN PCOS patients, glucose tolerance improves over time ${ }^{14}$.

Weight loss on ovarian function in PCOS: Obesity contributes substantially to PCOS. Changes in body weight loss has been found to be associated with spontaneous resumption of menses, considerable lowering of LH, free testosterone and insulin levels, and an improvement in the rates of ovulation and pregnancy outcomes ${ }^{10,15}$.

Ovarian ablative surgery for PCOS: Wedge resection as a modality of treatment was recom-mended by Stein and Leventhal. However in present times the same objective can be achieved through laparoscopic ovarian drilling with laser or monopolar cautery (LEOS). However the advisability of adopting this procedure is still controversial. A recent randomized 
trial found no difference in the pregnancy and miscarriage risks between ovarian surgery or gonadotropin induced ovulation in women showing resistance to earlier clomiphene therapy ${ }^{16}$. Multiple pregnancy rates are reduced in women treated with ovarian drilling. The benefits of this surgery may be temporary ${ }^{17}$. Ovarian drilling does not improve the metabolic abnormalities associated with PCOS (Lemieux 1999). It should be noted that greater the amount of damage to the surface of the ovary, the greater the risk of post operative peri-ovarian adhesion formation. The current trend is to apply the diathermy at $4-5$ sites in each ovary for $4-5$ secs each using $40 \mathrm{~W}$ coagulating current ${ }^{3}$.

Cosmetic management: Mechanical hair removal (shaving, plucking, waxing, depilatory creams, electrolysis and laser vaporization) methods are in common use. Concommittant medical therapy yields better long term results.

Potential long-term consequences of polycystic ovary syndrome

Definite / common consequences of PCOS

- Insulin resistance: Type-II diabetes

- Endometrial hyperplasia / atypia

- Gestational diabetes

- Sleep apnoea

\section{Possible consequences of PCOS}

- Hypertension

- Coronary heart disease

- Dyslipidemia

- ? $\uparrow$ Risk of ovarian cancer

- ? $\uparrow$ Risk of abortion

\section{Ovarian hyper-stimulation syndrome}

Ovarian hyper-stimulation syndrome (OHSS) is an uncommon, but potentially life threatening complication of ovarian stimulation by ovarian induction agents. Some degree of ovarian hyperstimulation occurs in all women who respond to ovulation induction but should be distinguished from the clinical entity of OHSS. This iatrogenic condition was first described following the use of gonadotropins ${ }^{18}$, but now most commonly seen following the use of super-ovulation regimens that are routinely used in assisted conception. Data from world registries of assisted conception estimated that 100,000 cycles occur annually resulting in 100-200 cases of severe OHSS every year worldwide ${ }^{19}$.

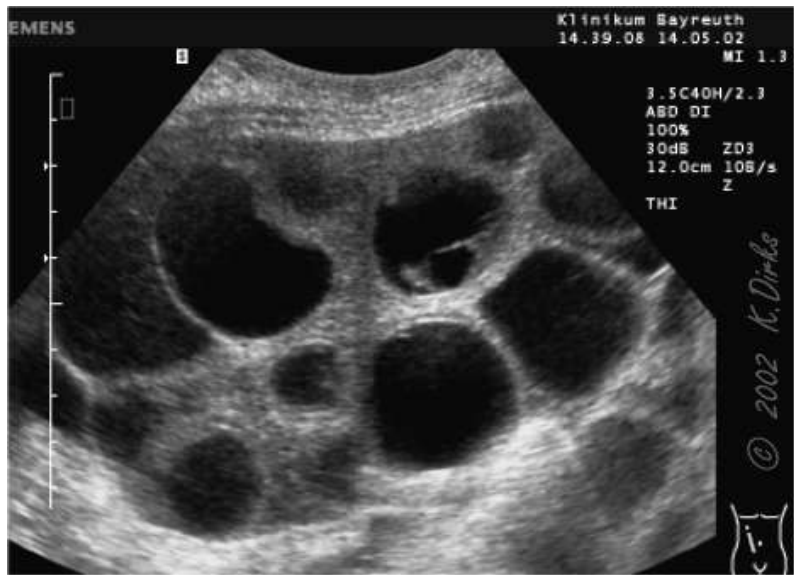

Figure 2. USG showing ovarian hyperstimulation.

\section{Pathophysiology}

According to the most widely accepted hypothesis, the basic pathophysiological event in OHSS is an increased capillary permeability resulting in intravascular fluid shift to the third space compartment. Many substances have been implicated in this pathogenesis, increasingly evidence supports for immune system activation mediated by release of cytokines and vasoactive substances of the renninangiotensin (R-A) system and vascular endothelial growth factor (VEGF).

The candidate factors for causation of OHSS are:

Estrogen
Renin-angiotensin system
Prostaglandins
Histamine
Prolactin
Cytokines
Vascular endothelial growth factor
Interleukins (IL-2, IL-6, IL-8)

Balen and colleagues ${ }^{3}$ presented evidence to support peripheral arteriolar vasodilatation as the primary event in the pathogenesis of OHSS. The homeostatic response to this vascular change occurs through baroreceptors within the renal tubular juxtaglomerular apparatus causing activation of the reninangiotensin-aldosterone system and sympathetic nervous system. These compensatory mechanisms by retaining sodium which contributes to the fluid shift to the extravascular space as shown in the figure. 


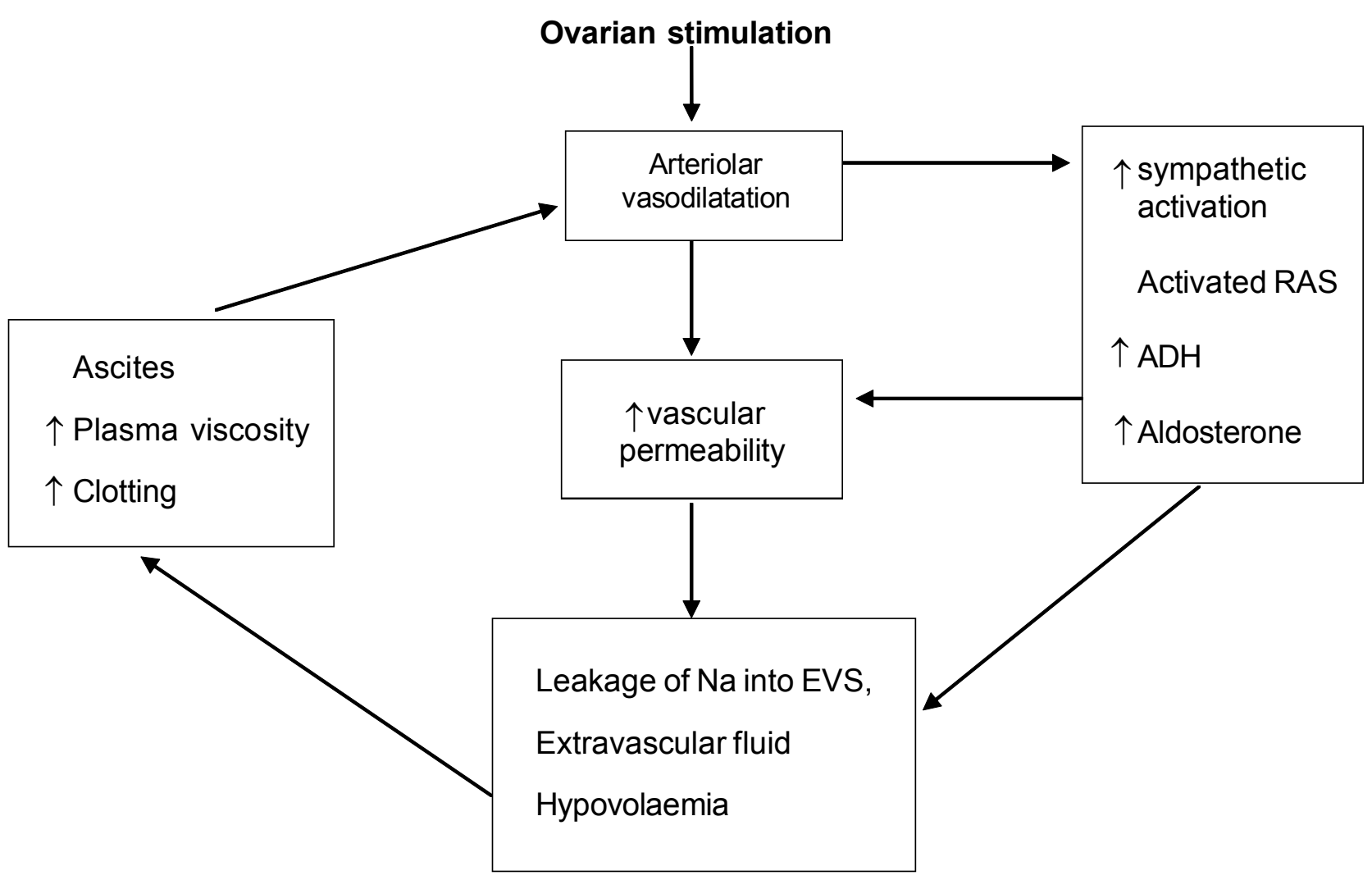

\section{Risk factors for OHSS}

1. Age younger patients

2. Low body weight

3. PCOS

4. High dose protocols

5. GnRH agonists

6. High absolute or rapidly rising serum estradiol levels

7. Number of follicles

8. Use of hCG

9. Luteal support

10. Previous episodes of OHSS

\section{Classification of OHSS}

There has been no unanimity in classifying OHSS. Various classifications for OHSS have been proposed $^{20,21}$ based on a variety of clinical ultrasound, biochemical and hematological parameters. A more simplified approach will help in the management decisions. Golan et al proposed the following classification that is most commonly followed.

\section{Mild OHSS}

Grade 1: abdominal distension and discomfort.
Grade 2: features of grade 1 plus nausea, vomiting and / or diarrhea; ovaries are enlarged to $5-12 \mathrm{~cm}$.

\section{Moderate OHSS}

Grade 3: features of mild OHSS plus ultrasonic evidence of ascites.

\section{Severe OHSS}

Grade 4: features of moderate OHSS plus clilnical evidence of ascites and/or hydrothorax and breathing difficulties.

Grade 5: all the above plus change in the blood volume, increased blood viscosity due to hemocon-centration, coagulation abnormality and diminished renal perfusion and function.

\section{Prevention of OHSS}

The trigger for OHSS is hCG and the only certain way of preventing this syndrome is to withhold its administration and cancel the cycle. Several measures have been advocated to reduce the possibility of OHSS in cases deemed to be at risk. None of these will eliminate OHSS, but, until the sensitivity of risk identification is increased, their use should be considered in high risk cases. 


\section{Reducing hCG stimulation and surrogate hCG}

hCG is the trigger for OHSS and the patient at risk should be given a reduced dose prior to oocyte recovery. Lewit et al ${ }^{22}$ demonstrated a complete avoidance of OHSS in their study using this technique but until further trials are undertaken it cannot be advocated in routine practice. In other cases use of $\mathrm{GnRh}$ agonist for trigger can also be considered.

\section{Coasting}

This approach involves delaying the administration of hCG for several days after the ovarian follicles have reached maturity during which time the plasma estradiol levels fall. Several case controlled studies $^{23,24}$ have reported the avoidance of severe complications, but not elimination of OHSS. Maximum window for delay is not clear and all studies show a reduction in egg yield.

\section{Elective cyopreservation of embryos}

This approach will prevent endogenous hCG source when a pregnancy occurs. This will limit OHSS by avoiding the pregnancy, at the same time maintaining the possibility of a pregnancy resulting from the replacement of thawed embryos in a subsequent non-stimulated cycle.

\section{Prophylactic albumin and substitutes}

Some randomized controlled trials have shown a significant reduction in the incidence of OHSS in at risk patients given prophylactic albumin at the time of egg collection. The rationale for the use of prophylactic albumin is to act as a carrier protein to prevent leakage of vasoactive substances in to extravascular space.

\section{Immunoglobulins}

Levels of $\operatorname{IgG}$ and $\operatorname{IgA}$ gammaglobulins in the plasma of patient with severe OHSS have been shown recently and with the use of IV immunoglobulin's the incidence of severe OHSS can be significantly reduced.

\section{Luteal phase support}

Pregnancy rates are higher with the use of hCG as luteal support as compared to progesterone but it increases the chances of OHSS. At present, progesterone appears to be the luteal phase support of choice. Duphaston, a dydrogesterone progesterone in oral doses of $30 \mathrm{mg}$ daily has shown to have better pregnancy rates as compared to vaginal micronised progesterone. In addition oral preparations have a better patient compliance than vaginal $u^{25}$.

\section{Use of cabergoline}

Cabergoline in the dose of $0.5 \mathrm{mg}$ daily for 4-7 days is very effective to reduce the incidence of OHSS. This is due to its anti dopaminist action which reduces the VGEF2 production, which is one of the initiators of OHSS.

\section{Treatment of established OHSS}

Management of established case should be undertaken under the supervision of a specialist in reproductive medicine. The most common complications of OHSS are thromoembolism, renal failure, ovarian torsion, intra-abdominal bleeding gastrointestinal symptoms, ARDS and liver dysfunction. The treatment is aimed at limiting these serous and life threatening complications the most common of which are thromboembolic. It should include the following:

Close monitoring of at risk patients

Low threshold for admission

Involve expert help at an early stage

Correct hypovolaemia with colloids

Prophylactic heparin

Drainage of 3rd space fluid accumulation

Avoid surgical intervention

\section{Conclusion}

In summary, PCOS is a heterogenous familial condition. Ovarian dysfunction is the main symptom. The ovary is influenced by factors including the gonadotrophins, insulin, growth factors, which are dependent upon both genetic and environmental influence. Long term risks include diabetes and cardiovascular disease.

Treatment has been symptomatic including the infertile subgroup. However better understanding of the pathogenesis and more in-depth knowledge of its genetic basis will make better treatment options available.

\section{References}

1. Fauser B, Tarlatzis B, Chang J, Azziz R, Legro R, Dewailly $\mathrm{D}$, et al. Rotterdam ESHRE/ASRM sponsored PCOS consensus workshop group. Revised 2003 consensus on diagnostic criteria and long term health risk related to PCOS. Hum Reprod 2004; 19: 41-7.

2. Franks S. Polycystic ovary syndrome. N Eng J Med 1995; 333: 853-86.

3. Balen AH, Conway GS, Homborg R, Legro RS. PCOS - A Quideto Clinical Management. London: Taylor \& Francis 2005. 
4. Rodin DA, Bano G, Bland JM, Taylor K, et al. PCOS and associated metabolic abnormalities in Indian sub continental asian women. Clin Endocrinology (Oxford) 1998; 49: 91-9.

5. Portsky L. On th paradox of insulin induced hyperandrogenism in insulin resistant states. Endocr Rev 1991; 12: 3-13.

6. Dunaif A. Insulin resistance and the polycystic ovary syndrome: mechanism and implications for pathogenesis. Endocr Rev 1997; 18(6): 774.

7. Urman B, Sarac E, Dagan L, et al. Pregnancy in infertile PCOD patients, complications and outcome. J Reprod Med 1997; 42: 501-5.

8. Anttila L, Karjala K, Panttila RA, et al. PCOS in women with gestational diabetes. Obstet Gynecol 1998; 92: 13-16.

9. Dahlgren E, Friberg LG, Johansson S, et al. Endometrial carcinoma, ovarian dysfunction - a risk factor in young women. Eur J Obstet Gynecol Reprod Biol 1991; 41: 143-50.

10. Clark AM, Thornley B, Tomlinson L, Norman RJ. Weight loss in obese infertile women results in improvement in reproductive outcome for all forms of fertility treatment. Human Reproduction 13: 1502-1505. Balasch J, Fabregues F, Arroya V. Peripheral arterial vasodialtition hypothesis: a new insight into the pathogeneisis of ovarian hyperstimulation syndrome. Hum Reprod 1998; 10: 2718-30.

11. British National Formulary. 51st Edition. London. British Medical Association and Royal Pharmaceutical Society of Great Britain 2006. P 380.

12. Biljan M, Hemmings R, Brassard N. The outcome of 150 babies following letrozole or letrozole and gonadotrophins. Annual Meeting of the American Society for Reproductive Medicine, Montral, Quebec 2005.

13. NICE Cilinical Guideline. 2004. Fertility: assessment and treatment for people with fertility problems. London: National Institute for Clinical Excellence.

14. Knowler WC, Barrett-Counor E, Fowler SE, et al. Reduction in the incidence of type 2 diabetes with lifestyle intervention or metformin. N Eng J Med 2002; 346: 393-403.
15. Kiddy DS, Hamilton Fairley D, Bush A, et al. Improvement in endocrine and ovarian function during dietary treatment of obese women with PCOS. Clin Endocriol 1992; 36: 105-11.

16. Farquhar CM, Williamson K, Gudex G, et al. A randomized controlled trial of laparoscopic ovarian diathermy versus gonadotropin therapy for women with clomiphene resistant PCOS. Fertil Steril 2002; 76: 404-11.

17. Donesky BW, Adashi EY. Surgically induced ovulation in PCOS, wedge resection revisited in the age of laparoscopy. Fertil Steril 1995; 63: 439-63.

18. Mazer C, Ravetz E. Effect of combined administration of chrionic gonadotrophin and pituitary synergist of on human ovary. Am J Obstet Gynecol 1941; 41: 474.

19. Schenker JG, Ezra Y. Complications of assisted reproduction techniques. Fertil Steril 1994; 61: 411-22.

20 Golan A, Ron-El R, Herman A, Sofer Y. Ovarian hyperstimulaion syndrome: an update dreview. Obstet Gynecol Surv 1989; 44: 430-40.

21. Navot D, Relou A, Birkenfeld A. Risk factor and prognostic variables in the ovarian hyperstimulation syndrome. Am J Obstet Gynecol 1998; 159: 210-15.

22. Lewit N, Kol S, Manor D, Itskovitz -Eldor J. Comparion of GnRG - a hCG for the induction of ovulation and prevention of ovarian hyperstimulaion syndrome: a case-control study. Hum Reprod 1996; 11: 1399-402.

23. Dhont M, Van der Straeten F, Sutter P. Prevention of severe hyperstimulation by coasting. Fertil Steril 1998; 5: 847-50.

24. Sher G, Souves C, Feinman M, Massarani G. Prolonged coasting: an effective method for prevention of severe ovarian hyperstimulation syndrome in patients undergoing in vitro fertilization. Human Reprod 1995; 10: 3107-9.

25. Patki AS, Pawar V. Modulating fertility outcome in assisted reproductive technologies by the use of dydrogesterone. Gynaecological Endocrinology 2007; 23 (S1): 1-5. 